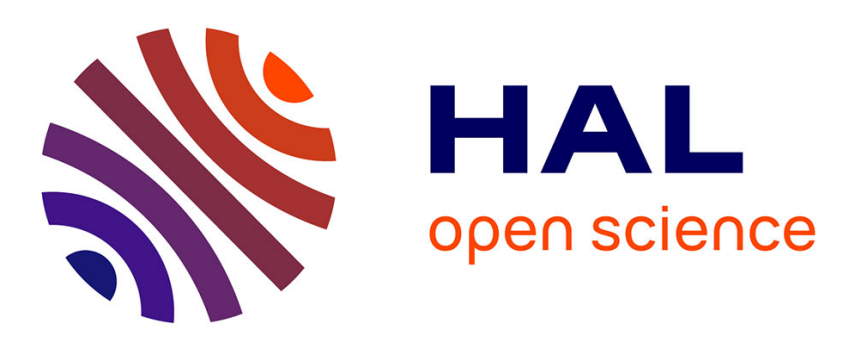

\title{
On the input-output approach towards distributed estimation
}

Fakhteh Saadatniaki, Anton Korniienko, Gérard Scorletti, Usman A. Khan

\section{To cite this version:}

Fakhteh Saadatniaki, Anton Korniienko, Gérard Scorletti, Usman A. Khan. On the input-output approach towards distributed estimation. ECC, Jun 2018, Limassol, Cyprus. 10.23919/ECC.2018.8550346 . hal-01984222

\section{HAL Id: hal-01984222 \\ https://hal.science/hal-01984222}

Submitted on 16 Jan 2019

HAL is a multi-disciplinary open access archive for the deposit and dissemination of scientific research documents, whether they are published or not. The documents may come from teaching and research institutions in France or abroad, or from public or private research centers.
L'archive ouverte pluridisciplinaire HAL, est destinée au dépôt et à la diffusion de documents scientifiques de niveau recherche, publiés ou non, émanant des établissements d'enseignement et de recherche français ou étrangers, des laboratoires publics ou privés. 


\title{
On the input-output approach towards distributed estimation
}

\author{
Fakhteh Saadatniaki ${ }^{\dagger}$, Anton Korniienko ${ }^{\ddagger}$, Gerard Scorletti ${ }^{\ddagger}$, and Usman A. Khan ${ }^{\dagger}$
}

\begin{abstract}
In this paper, we study distributed estimation of continuous-time, linear time-invariant systems monitored by a network of agents communicating over a graph. We assume that no agent may possess enough measurements in its neighborhood to estimate the entire state vector on its own. In this context, we provide a networked Kalman-type estimator that combines prediction and innovation with information fusion among the neighboring agents and consider an approach based on designing static estimator gains. The main contribution of this paper is to analyze the estimation error using the notions of dissipativity and the input-output approach, which enable us to formulate stability and performance arguments as quasiconvex optimization problems involving linear matrix inequalities. We show that the resulting estimation error is stable and further ensures a given level of performance regarding noise rejection. Simulations illustrate the concepts described in this paper.

Index Terms-Distributed control; Consensus control and estimation; Agents and autonomous systems
\end{abstract}

\section{INTRODUCTION}

Recently, there has been a significant amount of work in the area of distributed estimation of physical and social phenomena [1]-[3]. Some prime examples include tracking in multi-agent networks [4], [5], estimation or optimization in large-scale systems [6], [7], or studying opinion formation or voting models in complex social networks [8]. Estimating the underlying dynamics in such systems is a challenging problem as measurements are typically distributed over a network of geographically-dispersed agents (sensors, robots, individuals). Distributed estimation, see e.g., relevant work in [9]-[11], thus enables estimating the dynamics without collecting measurements at a central location, a practically infeasible task, but by enabling local estimation and interagent information exchange. In this paper, we focus on the distributed estimation of a Continuous-Time, Linear TimeInvariant (CT-LTI) dynamical system whose measurements are distributed over a network of sparsely-connected agents.

Relevant work on decentralized estimation of CT-LTI systems can be found in [12]-[15]. For instance, Ref. [12] considers decentralized state estimation of linear stochastic systems based on a combination of local Kalman filters and a dynamic consensus scheme among the agents. Ref. [13] proposes a theoretical framework for coupled distributed estimation and motion control of mobile sensor networks based on distributed Kalman filtering for collaborative target

${ }^{\dagger} \mathrm{FS}$ and UAK are with the Department of Electrical and Computer Engineering at Tufts University, Medford, MA 02155, USA, \{fakhteh.saadatniaki, khan@ece.\}@tufts.edu. His work is supported by an NSF Career award \# CCF-1350264.

${ }^{\ddagger} \mathrm{AK}$ and GS are with Laboratoire Ampère, Université de Lyon, École Centrale de Lyon, 69134 Ecully Cedex, France, ffirst name $\} .\{$ last name $\} @ e c-l y o n$. fr. Their work is supported by a grant from la Région Rhône-Alpes. tracking. Ref. [14] extends adaptive diffusion models to continuous-time setups. Moreover, a recursive algorithm is presented in [15] for the distributed estimation of a moving target under switching interconnection topologies, where the stability of the algorithm is analyzed under mild observability and connectivity assumptions. Of significant relevance to this paper is Ref. [16], which tackles the problem of control law design for interconnected systems, ensuring global stability and a certain pre-specified performance criteria using the notion of dissipativity, focusing on certain input-output properties of dynamical systems related to the conservation, dissipation, and transport of energy loosely referred to as dissipative properties [17].

In this paper, we assume that the no agent possesses enough measurements in its neighborhood to be able to estimate the entire state-vector on its own. In this sense, standard estimation techniques are not applicable and we propose a consensus plus innovation estimator that recovers the observability at each agent with the help of consensus on the neighboring predictions. To keep the estimator design simple and develop an estimator that requires minimal computation and coordination, we use a static gain that is same for both of the consensus and the innovation terms. In this context, we use the machinery developed in Ref. [16] and cast the distributed estimation problem in a similar decentralized control framework, where based on dissipativity characterization of the dynamical systems, we show that the gain design problem can be reduced to two quasiconvex optimization problems under Linear Matrix Inequality (LMI) constraints. In particular, we apply the bounded-real lemma and matrix elimination procedure to obtain existence conditions and a design procedure for a static estimator gain, which guarantees stable error dynamics while also satisfying a given performance level in terms of an upper bound on the $H_{\infty}$-norm of the global error transfer function. Our design is based on the input-output approach, i.e., given a power spectral density (PSD) specification on the input, the $H_{\infty}$-norm is closely related to the PSD of the output, see e.g., [18], [19].

We now describe the rest of the paper. Section II formulates the problem and describes related concepts on dissipativity and the input-output approach. Sections III and IV study the estimation error analysis and present our main results on the design of a static estimator gain ensuring stability and performance specifications, respectively. Section V illustrates our results and Section VI concludes the paper.

Notation: The superscripts ' $T$ ' and ' $*$ ' denote transpose and complex-conjugate transpose, respectively. The $N \times N$ identity matrix is denoted by $I_{N}$ and the $n \times m$ zero matrix is 
denoted by $0_{n \times m}$. The indexes are dropped if the context is clear. The diagonal aggregation of two matrices $A$ and $B$ is denoted by $\operatorname{diag}(A, B)$. With matrix $G$ partitioned into four blocks $G_{11}, G_{12}, G_{21}$, and $G_{22}, G \star K$ denotes the Redheffer product, [20], i.e.,

$$
G \star K=G_{11}+G_{12} K\left(I-G_{22} K\right)^{-1} G_{21} ;
$$

similarly, $K \star G=G_{22}+G_{21} K\left(I-G_{11} K\right)^{-1} G_{12}$. We use $\Re\{\alpha\}$ to denote the real part of a complex number $\alpha \in \mathbb{C}$. For a stable LTI system represented by transfer matrix $G,\|G\|_{\infty} \triangleq \sup \{\|G(j \omega)\| \mid \omega \in \mathbb{R}\}$ denotes its $H_{\infty}$-norm, [18]. The $i$ th eigenvalue of a real square matrix $P$ is denoted by $\lambda_{i}(P)$. For symmetric matrices, ' $\succeq$ ' (' $\succ$ ') denotes positive semi-definiteness (positivedefiniteness). Finally, $\mathbb{R}^{+}$represents the set of nonnegative real numbers.

\section{Problem Formulation}

Consider the following CT-LTI dynamics:

$$
\dot{\mathbf{x}}(t)=A \mathbf{x}(t), \quad t \geq 0,
$$

where $t$ is the continuous-time variable, $\mathbf{x}(t) \in \mathbb{R}^{n}$ is the state at time $t$, and $A$ is the system matrix. The measurements for this system are distributed over a network of $N$ agents, communicating according to a graph $\mathcal{G}=(\mathcal{V}, \mathcal{E})$, where $\mathcal{V}=\{1,2, \ldots, N\}$ is the set of agents and $\mathcal{E}$ is the set of edges (links) between the agents. The neighborhood at the $i$ th agent is defined as $\mathcal{N}_{i} \triangleq\{i\} \cup\{j \mid(i, j) \in \mathcal{E}\}$ and each agent $i$ has the following observation model:

$$
\mathbf{y}_{i}(t)=H_{i} \mathbf{x}(t)+\mathbf{r}_{i}(t),
$$

where $\mathbf{y}_{i}(t) \in \mathbb{R}^{p_{i}}$ is the local measurement, $H_{i}$ is the local observation matrix, and $\mathbf{r}_{i}(t)$ is the zeromean additive noise whose PSD is bounded by 1 . Letting $\mathcal{A} \in\{0,1\}^{N \times N}$ to denote the corresponding adjacency matrix and $D=\operatorname{diag}\left(\left|\mathcal{N}_{1} \backslash\{1\}\right|, \cdots,\left|\mathcal{N}_{N} \backslash\{N\}\right|\right)$ to denote the degree matrix, the normalized adjacency matrix is defined as $\overline{\mathcal{A}}=D^{-1} \mathcal{A}$.

Given the dynamical system described by Eq. (1) and local observations in Eq. (2), the goal is to estimate the state $\mathbf{x}(t)$ of the system in a distributed manner. We assume that the system is globally observable, i.e., with the system matrix, $A$, and the collection of all measurement matrices, $H_{i}$ 's. However, no agent is assumed to be observable in its neighborhood, i.e., the system matrix, $A$, is not necessarily observable with the measurement matrices, $H_{j}, j \in \mathcal{N}_{i}$, at any agent in the network. Clearly, due to a lack of (neighborhood) observability, no agent can implement the standard Kalman filtering equations to estimate the state, $\mathbf{x}(t)$, even if it collects all of the measurements in its neighborhood. The distributed estimation problem is thus to estimate the state of the system in this setting. Finally, we note that there is no process noise in Eq. (1). Nevertheless, the state estimation problem considered here is of relevance because the initial conditions are not known, the measurements are corrupted by noise, and no agent is locally observable.
We propose a variant of the Networked Kalman-type Estimator (NKE), initially introduced in [21], which follows a prediction + innovation framework based on state and output exchange among agents with an additional consensus term from inter-agent interactions to address the distributed nature of the problem. Not only does this force agreement but it also diffuses information on the state variables for which there may be no measurement in the neighborhood:

$$
\begin{aligned}
\dot{\widehat{\mathbf{x}}}_{i}(t) & =\underbrace{A \widehat{\mathbf{x}}_{i}(t)}_{\text {predictor }}+\underbrace{\sum_{j \in \mathcal{N}_{i}} K_{i j}\left(\widehat{\mathbf{x}}_{j}(t)-\widehat{\mathbf{x}}_{i}(t)\right)}_{\text {state exchange: consensus }} \\
& +\underbrace{\sum_{j \in \mathcal{N}_{i}} B_{i j} H_{j}^{\top}\left(\mathbf{y}_{j}(t)-H_{j} \widehat{\mathbf{x}}_{i}(t)\right)}_{\text {measurement exchange: innovation }},
\end{aligned}
$$

where $\widehat{\mathbf{x}}_{i}(t)$ is the local state estimate at agent $i, K_{i j} \in \mathbb{R}^{n \times n}$ are consensus gain matrices, and $B_{i j} \in \mathbb{R}^{n \times n}$ are innovation gain matrices between agents $i$ and $j$. Since each agent estiamtes the same dynamical system, we assume the gain matrices to be constant over the consensus and innovation terms and across each agent, i.e., $K_{i j}=\frac{K}{\left|\mathcal{N}_{i} \backslash\{i\}\right|}, B_{i j}=K$, where $K \in \mathbb{R}^{n \times n}$; this also serves as a baseline with respect to the solutions obtained with higher degrees of freedom.

\section{A. Error Dynamics}

Defining the local estimation error as $\mathbf{e}_{i}(t) \triangleq \widehat{\mathbf{x}}_{i}(t)-\mathbf{x}(t)$, it is straightforward to show that

$$
\begin{aligned}
\dot{\mathbf{e}}_{i}(t) & =\dot{\hat{\mathbf{x}}}_{i}(t)-\dot{\mathbf{x}}(t) \\
& =A \mathbf{e}_{i}(t)+\frac{K}{\left|\mathcal{N}_{i} \backslash\{i\}\right|} \sum_{j \in \mathcal{N}_{i}}\left[\mathbf{e}_{j}(t)-\mathbf{e}_{i}(t)\right] \\
& -K\left(\sum_{j \in \mathcal{N}_{i}} H_{j}^{\top} H_{j}\right) \mathbf{e}_{i}(t)+\underbrace{K \sum_{j \in \mathcal{N}_{i}} H_{j}^{\top} \mathbf{r}_{j}(t)}_{\text {external input due to noise }} .
\end{aligned}
$$

The matrix $K$ can be thought of as a static estimator gain, designing which is the main goal of this paper such that the estimation error is stable at each agent and meets certain performance criteria with regard to noise rejection. The block diagram corresponding to the graphical representation of the local estimation error dynamics is depicted in Fig. 1.

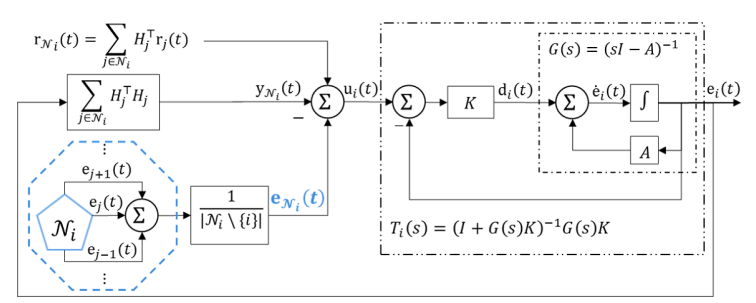

Fig. 1: Local error dynamics

The signals enclosed in the dashed octagon represent the estimation errors from the neighborhood $\mathcal{N}_{i}$ of agent $i$, constructing the auxiliary variable $\mathbf{e}_{\mathcal{N}_{i}}(t)$ as labeled in Fig. 1. The aggregate auxiliary signal from the entire network, 
denoted as $\overline{\mathbf{e}}(t)$, reveals how the network topology affects the global estimation error dynamics and is given by

$$
\overline{\mathbf{e}}(t)=\left[\begin{array}{c}
\mathbf{e}_{\mathcal{N}_{1}}(t) \\
\vdots \\
\mathbf{e}_{\mathcal{N}_{N}}(t)
\end{array}\right]=\left(\overline{\mathcal{A}} \otimes I_{n}\right)\left[\begin{array}{c}
\mathbf{e}_{1}(t) \\
\vdots \\
\mathbf{e}_{N}(t)
\end{array}\right] \triangleq\left(\overline{\mathcal{A}} \otimes I_{n}\right) \mathbf{e}(t) .
$$

Next we investigate the effect of agent measurements on the global estimation error dynamics:

$$
\overline{\mathbf{y}}(t)=\left[\begin{array}{c}
\mathbf{y}_{\mathcal{N}_{1}}(t) \\
\vdots \\
\mathbf{y}_{\mathcal{N}_{N}}(t)
\end{array}\right]=D_{H}\left[\begin{array}{c}
\mathbf{e}_{1}(t) \\
\vdots \\
\mathbf{e}_{N}(t)
\end{array}\right]
$$

where $D_{H}$ is the block-diagonal matrix with $\sum_{j \in \mathcal{N}_{i}} H_{j}^{\top} H_{j}$ being its $i$ th block, and

$$
\overline{\mathbf{r}}(t)=\left[\begin{array}{c}
\mathbf{r}_{\mathcal{N}_{1}}(t) \\
\vdots \\
\mathbf{r}_{\mathcal{N}_{N}}(t)
\end{array}\right]=\left[\begin{array}{c}
\sum_{j \in \mathcal{N}_{1}} H_{j}^{\top} r_{j}(t) \\
\vdots \\
\sum_{j \in \mathcal{N}_{N}} H_{j}^{\top} r_{j}(t)
\end{array}\right],
$$

which is the external input due to measurement noise.

Note that $T_{i}(s)$ (as labeled in Fig. 1) can be rewritten as $G(s) K(I+G(s) K)^{-1}$ based on the push-through rule from [19, Chapter 3]; consequently, we can express $T_{i}(s)$ in terms of the Redheffer product $\mathcal{P}(s) \star K$ with

$$
\mathcal{P}(s)=\left[\begin{array}{ll}
\mathcal{P}_{11}(s) & \mathcal{P}_{12}(s) \\
\mathcal{P}_{21}(s) & \mathcal{P}_{22}(s)
\end{array}\right]=\left[\begin{array}{rr}
\mathbf{0} & G(s) \\
I & -G(s)
\end{array}\right] .
$$

For the global error process, concatenating $\mathbf{u}_{i}(t)$ (as labeled in Fig. 1) in a vector $\mathbf{u}(t)$ results in the internal signal

$$
\mathbf{u}(t)=\overline{\mathbf{r}}(t)+\overline{\mathbf{e}}(t)-\overline{\mathbf{y}}(t)=\overline{\mathbf{r}}(t)+\left[\left(\overline{\mathcal{A}} \otimes I_{n}\right)-D_{H}\right] \mathbf{e}(t) .
$$

The resulting global error process with $\overline{\mathbf{r}}(t)$ as the input and $\mathbf{e}(t)$ as the output is represented in Fig. 2. This system can be interpreted as the interconnection $F$ of $N$ identical subsystems $T_{i}(s)=T(s)$, mathematically denoted as $\left(I_{N} \otimes T(s)\right) \star F$, where $\mathbf{e}(s)=\left(I_{N} \otimes T(s)\right) \mathbf{u}(s)$. As indicated in Fig. 2, the interconnection $F$ is static; i.e., a gain matrix, which means that the relation [in Fig. 2] holds in both time and frequency domains. We now describe the main problem addressed in this paper.
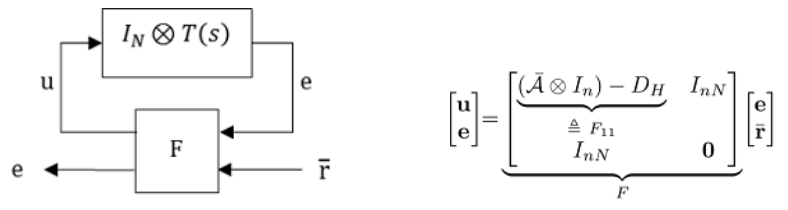

Fig. 2: Global error dynamics

Problem 1: (Distributed Estimation): Given the above formulation, find the estimator gain $K$ such that given the local dynamics $\mathcal{P}$ as defined in Eq. (8) and the interconnection matrix $F$ in Fig. 2, the corresponding global error dynamics is stable and satisfies the performance specification

$$
\left\|\left(I_{N} \otimes T(s)\right) \star F\right\|_{\infty}<\eta,
$$

for a given $\eta>0$. This objective directly affects noise rejection properties of the global error by setting an upper bound $\eta$ on the noise amplification from $\overline{\mathbf{r}}$ to e, see Fig. 2. To this aim, we will design procedures based on the dissipativity properties of the interconnection matrix $F$ and the local system $T(s)$. The advantage of the dissipativity framework lies in the fact that it reduces the complexity of global stability and performance analyses by studying the local [sub]systems, which is the main motivation behind inputoutput approaches in the study of networked systems, see e.g., [22]-[24]. The analysis is carried out in Sections III and IV for stability and performance, respectively. Preliminaries and essential results related to dissipativity are described next.

\section{B. Preliminaries on Input-output Approach}

Consider a general state space

$$
\begin{array}{ll}
\dot{\mathbf{x}}=f(\mathbf{x}, \mathbf{u}), & \mathbf{u} \in \mathcal{U}=\mathbb{R}^{m}, \\
\mathbf{y}=h(\mathbf{x}, \mathbf{u}), & \mathbf{y} \in \mathcal{Y}=\mathbb{R}^{p},
\end{array}
$$

where $\mathbf{x} \in \mathbb{R}^{n}$ is the state and $(\mathbf{u}, \mathbf{y})$ is the input-output pair. The concept of dissipativity describes the system by relating the internally stored energy of the system to a generalized energy supply rate function $s(\mathbf{u}(t), \mathbf{y}(t))$. There are many different notions of dissipativity introduced in various publications, see e.g., [17, Chapter 4] for a detailed discussion. In our analysis of distributed estimation, we use the following notion of dissipativity in frequency domain from [16]:

Definition 1 (Dissipativity): A causal, stable LTI system $\mathcal{T}$ is [strictly] $(X, Y, Z)$-dissipative, where $X=X^{\top}, Y$, and $Z=Z^{\top}$ are real matrices of appropriate dimensions such that

$$
\left[\begin{array}{cc}
X & Y \\
Y^{\top} & Z
\end{array}\right]
$$

is full-rank, if there exists $\varepsilon=0[>0]$ such that

$$
\left[\begin{array}{c}
I \\
\mathcal{T}(j \omega)
\end{array}\right]^{*}\left[\begin{array}{cc}
X & Y \\
Y^{\top} & Z
\end{array}\right]\left[\begin{array}{c}
I \\
\mathcal{T}(j \omega)
\end{array}\right]+\varepsilon I \preceq 0 .
$$

for almost every $\omega \in \mathbb{R}^{+}$.

The following result from [16] serves as the basis for our proposed solution to the problem of distributed estimation explained in the next two sections.

Theorem 1: Given $\eta>0$, a stable interconnection $F$, a local plant $\mathcal{P}(s)$, and the real matrices $X=X^{\top} \prec 0, Y$, and $Z=Z^{\top}$ of appropriate dimensions, if there exist

(i) a positive-definite matrix $S \in \mathbb{R}^{N \times N}$ such that $F$ is $\left\{\operatorname{diag}\left(S \otimes X,-\eta^{2} I\right), \operatorname{diag}(S \otimes Y, 0), \operatorname{diag}(S \otimes Z, I)\right\}$ dissipative, and

(ii) a local controller $K$ such that $T(s)=\mathcal{P}(s) \star K$ is strictly $\left\{-Z,-Y^{\top},-X\right\}$-dissipative,

then the large-scale system $\left(I_{N} \otimes T(s)\right) \star F$, depicted in Fig. 2 is stable and $\left\|\left(I_{N} \otimes T(s)\right) \star F\right\|_{\infty} \leq \eta$.

Theorem 1 can be specialized to analyze only the internal stability by slightly modifying condition (i) as below.

(i') a positive-definite matrix $S$ such that $M=F_{11}$, defined in Fig. 2, is $\{S \otimes X, S \otimes Y, S \otimes Z\}$-dissipative. 
In this paper, we assume diagonal matrices of the form

$$
X=x I_{n}, \quad Y=y I_{n}, \quad Z=z I_{n},
$$

for the choice of $X, Y$, and $Z$ in Theorem 1 [and its modified version] and find $x, y$, and $z$ such that conditions (i) $\left[\left(\mathrm{i}^{\prime}\right)\right]$ and (ii) are both satisfied. Matrices of more general structures will be the focus of future studies.

\section{ERROR STABILITY}

Recall that our goal is to design the estimator gain matrix $K$ such that the global error dynamics are internally stable and satisfy a given level of performance. In this section, we consider the stability of the error process, which ensures that the estimation error would vanish if no measurement noise is present across the network; the performance will be discussed in the next section. We only focus on the case where the system matrix $A$ is not Hurwitz since otherwise, the error process is stable with $K=\mathbf{0}_{n}$. We now present our main result on the error stability.

Theorem 2 (Stability): Given the dynamics in Eq. (1) with non-Hurwitz system matrix $A$, the global estimation error process $\left(I_{N} \otimes T(s)\right) \star F$, and $M=F_{11}=\overline{\mathcal{A}} \otimes I_{n}-D_{H}$, there exists an estimator gain matrix $K \in \mathbb{R}^{n \times n}$ ensuring internal stability if there exist a positive-definite matrix $S \in \mathbb{R}^{N \times N}$, and real values $\chi>0$ and $c$ such that

$$
\begin{gathered}
M^{\top}(\underbrace{S \otimes I_{n}}_{\triangleq \widetilde{S}}) M \preceq \chi\left(c M-I_{n N}\right)^{\top} \widetilde{S}\left(c M-I_{n N}\right), \\
\chi<\min \left\{\frac{1}{c^{2}}, \frac{1}{(c-1)^{2}}\right\} .
\end{gathered}
$$

The proof of Theorem 2 is beyond the scope of this paper and is omitted because of space limitations. We have included a sketch of the proof here.

Proof Sketch : Directly applying the modified version of Theorem 1 and choosing $x=-1, y=c$, and $z=\frac{1}{\chi}-c^{2}$ in Eq. (13), Eq. (14) is equivalent to condition (i'). Moreover, condition (ii) in Theorem 1 can be expressed as

$$
\chi(T(j \omega)-c I)^{*}(T(j \omega)-c I)-I \prec 0,
$$

which is equivalent to $\|\widehat{T}\|_{\infty}<1$ for the auxiliary system $\widehat{T}(s)=\sqrt{\chi}(T(s)-c I)$. Applying Bounded-Real Lemma [25, Chapter 10] to the state-space representation of $\widehat{T}(s)$ and introducing the change of variables $Q=P K$, $\|\widehat{T}\|_{\infty}<1$ if and only if there exists a positive-definite matrix $P \in \mathbb{R}^{n \times n}$ such that

$$
\left[\begin{array}{cc}
A^{\top} P+P A-\left(Q^{\top}+Q\right)+\chi I & Q-c \chi I \\
Q^{\top}-c \chi I & \left(c^{2} \chi-\gamma^{2}\right) I
\end{array}\right] \prec 0,
$$

for $\gamma^{2}=1$. The proof is completed by deriving the equivalence between Eq. (15) and the feasibility of Eq. (17) through the application of the Elimination Procedure for Matrix Variables [25, Chapter 2] on Eq. (17).

Theorem 2 establishes the existence conditions for a stabilizing estimator gain matrix $K$. In the remainder of this section, we provide an algorithm to compute $K$ based on this theorem and the resulting observations made in the following Remarks.

Remark 1: In the case of a single-input and single-output system $T$, Eq. (16) means that the Nyquist plot of the transfer function $T(s)$ lies inside the circle centered at $c+j 0$ with radius $\frac{1}{\sqrt{\chi}}$. Similar interpretation applies to the multiple-input and multiple-output case as well. To relax this constraint for a given center $c$, the radius has to be maximized, which is equivalent to minimizing $\chi$.

The key idea of the algorithm is based on the fact that minimizing $\chi$, as mentioned in Remark 1, would strengthen the constraint in Eq. (14); therefore, Eqs. (14) and (16) cannot be simultaneously relaxed. As a result, the following optimization problem can be solved to satisfy the conditions of Theorem 2, leading to the computation of the estimator gain matrix $K$ ensuring global stability.

$$
\begin{aligned}
& \underset{\chi, S}{\operatorname{minimize}} \quad \chi \\
& \text { subject to } \quad S \succ 0, \widetilde{S}=S \otimes I_{n}, \\
& \qquad M^{\top} \widetilde{S} M \preceq \chi\left(c M-I_{n N}\right)^{\top} \widetilde{S}\left(c M-I_{n N}\right),
\end{aligned}
$$

which is a generalized eigenvalue problem for a fixed value of $c$ (see Remark. 2), with $S \in \mathbb{R}^{N \times N}$ and $\chi \in \mathbb{R}^{+}$as the decision variables, [25].

Remark 2: The center $c$ has to be fixed in order to have a tractable optimization problem in Eq. (18); it must be chosen in a way that Eq. (14) is feasible. The choice of $c$ can be based on the spectrum of $M$; in fact, it can be shown that Eq. (14) is feasible as long as

$$
|c| \leq \frac{1}{2 \max _{i}\left(\left|\Re\left\{\lambda_{i}(M)\right\}\right|\right)} .
$$

Remark 3: Having formulated the optimization in Eq. (18) as a generalized eigenvalue problem, we cannot include Eq. (15) as an explicit constraint; consequently, we proceed in the following iterative manner: Denoting the values obtained from the above procedure as $c^{*}$ and $\chi^{*}$, we use these values to verify whether the constraint in Eq. (15) is met, in which case the static estimator gain is computed as $K=\left(P^{\mathrm{f}}\right)^{-1} Q^{\mathrm{f}}$, where $P^{\mathrm{f}}$ and $Q^{\mathrm{f}}$ are solutions to the feasibility problem in Eq. (17) for $\gamma=1, c=c^{*}$, and $\chi=\chi^{*}$. If not, we fix a new value for $c$ from the interval obtained in Eq. (19) and repeat the earlier steps. Given a sufficiently large number of samples from the interval in Eq. (19) (gathered in the sample set $\mathcal{C}$ ), one approach is to do a linear search on $c$ until the conditions of Theorem 2 are met or the sample set is exhausted, the latter implying that a static estimator gain $K$, ensuring stability of the global error, was not found.

The above procedure to compute the static estimator gain is summarized in Algorithm 1.

Remark 4: In case of Hurwitz (stable) system matrix $A$, the condition in Eq. (15) simplifies to $\chi<\frac{1}{c^{2}}$. 


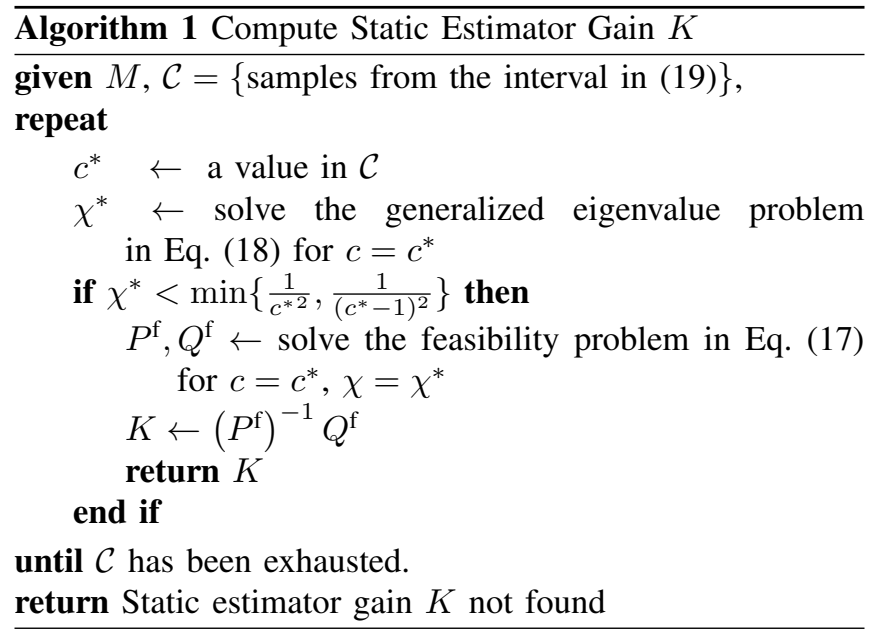

\section{PERFORMANCE}

In this section, we present our main result on $H_{\infty}$ performance characteristics of the global error process and compute estimator gain matrix $K$ satisfying the $H_{\infty}$ performance objective specified in Eq. (10) in addition to stability.

Theorem 3 (Performance): Given the global error process $\left(I_{N} \otimes T(s)\right) \star F$, there exists an estimator gain matrix $K$ ensuring performance objective $\left\|\left(I_{N} \otimes T(s)\right) \star F\right\|_{\infty}<\eta$, if there exist a positive-definite matrix $S \in \mathbb{R}^{N \times N}$, and real values $\chi>0$ and $c$ such that

$$
\Phi^{\top}\left[\begin{array}{cc:cc}
0 & 0 & 0 & 0 \\
0 & 0 & 0 & 0 \\
\hdashline 0 & 0 & \frac{1}{\chi} \widetilde{S} & 0 \\
0 & 0 & 0 & I
\end{array}\right] \Phi \preceq \chi \Phi^{\top}\left[\begin{array}{cc:cc}
\frac{1}{\chi} \widetilde{S} & 0 & 0 & 0 \\
0 & \frac{\eta^{2}}{\chi} & 0 & 0 \\
\hdashline 0 & 0 & 0 & 0 \\
0 & 0 & 0 & 0
\end{array}\right] \Phi,
$$

where $\widetilde{S} \triangleq S \otimes I_{n}, \Phi=\left[\begin{array}{cc:cc}I & 0 & -c I & 0 \\ 0 & I & 0 & 0 \\ \hdashline 0 & 0 & I & 0 \\ 0 & 0 & 0 & I\end{array}\right]\left[\begin{array}{l}I \\ F\end{array}\right]$, and

$$
\chi<\min \left\{\frac{1}{c^{2}}, \frac{1}{(c-1)^{2}}\right\} \text {. }
$$

Proof Sketch: The proof is based on Theorem 1 and follows the same procedure as the proof of Theorem 2 .

Similar to the case of internal stability, we present a systematic procedure to compute an estimator gain matrix $K$ satisfying the performance specification in Eq. (10) in addition to internal stability based on Theorem 3. An analogous observation to the one made in Remark 1, reveals that condition (ii) in Theorem 1 (equivalent to Eq.(16)) and Eq. (20) cannot be simultaneously relaxed, leading to the following optimization problem:

$$
\begin{array}{ll}
\underset{\chi, S}{\operatorname{minimize}} & \chi \\
\text { subject to } & S \succ 0, \widetilde{S}=S \otimes I_{n},
\end{array}
$$

$$
\Phi^{\top}\left[\begin{array}{cc:cc}
0 & 0 & 0 & 0 \\
0 & 0 & 0 & 0 \\
\hdashline 0 & 0 & \frac{1}{\chi} \widetilde{S} & 0 \\
0 & 0 & 0 & I
\end{array}\right] \Phi \preceq \chi \Phi^{\top}\left[\begin{array}{cc:cc}
\frac{1}{\chi} \widetilde{S} & 0 & 0 & 0 \\
0 & \frac{\eta^{2}}{\chi} & 0 & 0 \\
\hdashline 0 & 0 & 0 & 0 \\
0 & 0 & 0 & 0
\end{array}\right]
$$

with $\Phi$ as defined earlier.

To formulate the optimization in Eq. (22) as a generalized eigenvalue problem, we first solve the stability problem. Using the resulting values for $c, \chi$ and $\left\|\left(I_{N} \otimes T(s)\right) \star F\right\|_{\infty}$ (as a baseline for $\eta$ ), we introduce the change of variable $\varsigma=\frac{1}{\chi} S$ and parameter $\beta=\frac{\eta^{2}}{x}$. The resulting optimization problem, presented in Eq. (23), is a generalized eigenvalue problem with $\varsigma \in \mathbb{R}^{N \times N}$ and $\chi \in \mathbb{R}^{+}$as the decision variables, [25].

$$
\begin{aligned}
& \underset{\chi, \varsigma}{\operatorname{minimize}} \chi \\
& \text { subject to } \varsigma \succ 0, \widetilde{\varsigma}=\varsigma \otimes I_{n}, \\
& \Phi^{\top}\left[\begin{array}{ccccc}
0 & 0 & 0 & 0 \\
0 & 0 & 0 & 0 \\
\hdashline 0 & 0 & \widetilde{\varsigma} & 0 \\
0 & 0 & 0 & I
\end{array}\right] \Phi \preceq \chi \Phi^{\top}\left[\begin{array}{cc:cc}
\widetilde{\varsigma} & 0 & 0 & 0 \\
0 & \beta I & 0 & 0 \\
\hdashline 0 & 0 & 0 & 0 \\
0 & 0 & 0 & 0
\end{array}\right] \Phi .
\end{aligned}
$$

In the optimization problem above, $\beta$ acts as a tuning parameter; if the optimization problem has no solution, $\beta$ should be increased to relax the last constraint of the problem. Also note that for a given value of $\beta$, minimizing $\chi$ results in the minimization of the upper bound on noise amplification gain $\eta$ according to the relation $\eta^{2}=\chi \beta$.

\section{Simulations}

In this section, we illustrate the concepts introduced in this paper and evaluate the effectiveness of the proposed static gain distributed estimator in terms of internal stability and $H_{\infty}$ performance, experimentally. We consider a network of $N=20$ agents communicating over an undirected graph based on a 3-circulant graph with a few more links generated at random. The agents' goal is to estimate the state of an unstable $n=3$-dimensional CT-LTI dynamical system evolving according to the model in Eq. (1) with a randomly chosen initial condition $\mathbf{x}(0)$ using Eq. (3). We have considered a bare minimum measurement setup where 3 non-neighboring agents $i_{1}, i_{2}$, and $i_{3}$ are selected at random such that each of these three agents measures one of the system states in a mutually exclusive and collectively exhaustive manner while the rest make no measurement. Note that in this setup, observability is nonexistent from both individual and neighborhood perspectives.

In the remainder of this section, we demonstrate the stability and performance characteristics of the static gain estimator through a series of Monte Carlo simulations.

\section{A. Internal Stability}

To illustrate the stability of the estimaion error process, agents are assumed to have perfect (noise-free) observations; i.e., $\mathbf{r}_{i}(t)=0$. Given zero initial estimate $\widehat{\mathbf{x}}_{i}(0)$, each agent makes an estimate of the system state using Eq. (3) 
for $t \in[0,10]$ seconds. This procedure is repeated over 100 Monte Carlo simulations, each with randomly generated sets of non-Hurwitz system matrix $A$, communication graph $\mathcal{G}$, and observer agents $\left\{i_{1}, i_{2}, i_{3}\right\}$. Consistent with the theory, the distributed estimator in Eq. (3) results in asymptotically stable error as illustrated in Fig. 3a, where the normalized 2-norm of the global error, over each trial and averaged over 100 Monte Carlo simulations, has been plotted.

\section{B. Performance}

In this subsection, the performance level $\eta$ in Eq. (10) is set equal to 11 and the performance characteristics of the global error process are demonstrated in presence of noise signal $\overline{\mathbf{r}}(t)$ defined in Eq. (7), for the case where agent observations are subject to Additive White Gaussian Noise with PSD equal to $\sigma^{2}=0.25$. This procedure is repeated over 100 Monte Carlo simulations with a fixed set of system matrix $A$, communication graph $\mathcal{G}$, and the bare minimum observation scheme explained above. The resulting normalized 2-norm of the global error signal is plotted in Fig. 3b, averaged over 100 Monte Carlo simulations. We note that the error process is stable and the $H_{\infty}$-norm of the resulting global error process $\left\|\left(I_{N} \otimes T(s)\right) \star F\right\|_{\infty}$ is 10.6803 , satisfying the performance specification in Eq.(10).

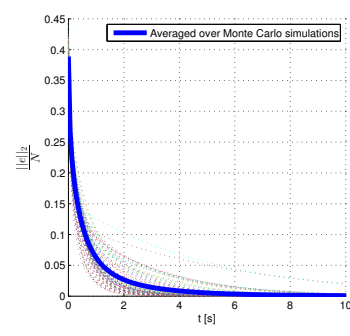

(a) Internal stability problem.

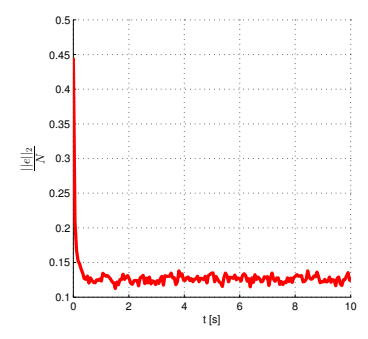

(b) Performance problem.
Fig. 3: Normalized error 2-norm $\frac{\|\mathbf{e}(t)\|_{2}}{N}$.

\section{CONCLUSIONS}

In this paper, we describe a static estimator gain design method to solve the distributed estimation problem for CTLTI systems monitored by a network of agents. In this context, we express the problem of distributed estimation as corresponding optimization problems and apply techniques based on the input-output approach to perform stability and performance analyses. We derive existence conditions for a static estimator gain matrix resulting in stable error dynamics, i.e., estimation error vanishing over time in the absence of measurement noise; additionally, we derive existence conditions regarding static estimator gain design, guaranteeing desirable performance characteristics for the error process in terms of noise rejection. The approach described in this paper serves as the foundation of future investigation towards extending the current work to dynamic estimator design meeting certain frequency-dependent performance objectives in addition to stability and noise rejection.

\section{REFERENCES}

[1] U. A. Khan and A. Jadbabaie, "On the stability and optimality of distributed Kalman filters with finite-time data fusion," in Proc. Am. Control Conf., Jun. 2011, pp. 3405-3410.

[2] S. Park and N. C. Martins, "Design of distributed LTI observers for state omniscience," IEEE Trans. Autom. Control, vol. 62, no. 2, pp. 561-576, Feb. 2017.

[3] M. Doostmohammadian and U. A. Khan, "Graph-theoretic inference in social networks," IEEE J. Sel. Topics Signal Process., vol. 8, no. 4, pp. 613-623, Aug. 2014.

[4] S. Oh, L. Schenato, P. Chen, and S. Sastry, "Tracking and coordination of multiple agents using sensor networks: System design, algorithms and experiments," Proc. IEEE, vol. 95, no. 1, pp. 234-254, Jan. 2007.

[5] S. Safavi and U. A. Khan, "An opportunistic algorithm for localization in mobile networks," IEEE Trans. Rob., vol. 33, no. 4, pp. 875-888, Aug. 2017.

[6] U. A. Khan, M. D. Ilić, and J. M. F. Moura, "Cooperation for aggregating complex electric power networks to ensure system observability," in Proc. 1st Int. Conf. on Infrastructure Syst. and Services: Building Networks for a Brighter Future (INFRA), Nov. 2008, pp. 1-6.

[7] C. Xi, R. Xin, and U. A. Khan, "ADD-OPT: Accelerated distributed directed optimization," IEEE Trans. Autom. Control, Aug. 2017, in press.

[8] M. A. Rahimian and A. Jadbabaie, "Group decision making and social learning," in Proc. 55th IEEE Conf. Decis. Control, Dec. 2016, pp. 6783-6794.

[9] D. W. Casbeer and R. Beard, "Distributed information filtering using consensus filters," in Proc. Am. Control Conf., Jun. 2009, pp. 18821887.

[10] U. A. Khan and A. Jadbabaie, "Collaborative scalar-gain estimators for potentially unstable social dynamics with limited communication," Automatica, vol. 50, no. 7, pp. 1909 - 1914, Jul. 2014.

[11] G. Battistelli and L. Chisci, "Kullback-Leibler average, consensus on probability densities, and distributed state estimation with guaranteed stability," Automatica, vol. 50, no. 3, pp. 707 - 718, Mar. 2014.

[12] S. S. Stankovic, M. S. Stankovic, and D. M. Stipanovic, "Decentralized parameter estimation by consensus based stochastic approximation," IEEE Trans. Autom. Control, vol. 56, no. 3, pp. 531-543, Mar. 2011.

[13] R. Olfati-Saber and P. Jalalkamali, "Coupled distributed estimation and control for mobile sensor networks," IEEE Trans. Autom. Control, vol. 57, no. 10, pp. 2609-2614, Oct. 2012.

[14] V. H. Nascimento and A. H. Sayed, "Continuous-time distributed estimation," in Conf. Rec. 45th Asilomar Conf. Signals, Syst., Comput., Nov. 2011, pp. 1761-1765.

[15] Z. Zhou, H. Fang, and Y. Hong, "Distributed estimation for moving target based on state-consensus strategy," IEEE Trans. Autom. Control, vol. 58, no. 8, pp. 2096-2101, Aug. 2013.

[16] A. Korniienko, G. Scorletti, E. Colinet, and E. Blanco, "Performance control for interconnection of identical systems: Application to PLL network design," Int. J. Robust Nonlin., vol. 26, no. 1, pp. 3-27, Jan. 2016.

[17] B. Brogliato, R. Lozano, B. Maschke, and O. Egeland, Dissipative Systems Analysis and Control: Theory and Applications, Communications and Control Engineering. Springer London, 2006.

[18] K. Zhou and J. C. Doyle, Essentials of robust control, Prentice Hall, Englewood Cliffs, NJ, 1997.

[19] S. Skogestad and I. Postlethwaite, Multivariable Feedback Control: Analysis and Design, Wiley, 2005.

[20] J. Doyle, A. Packard, and K. Zhou, "Review of LFTs, LMIs, and $\mu$," in Proc. 30th IEEE Conf. Decis. Control, Dec. 1991, pp. 1227-1232.

[21] U. A. Khan, S. Kar, A. Jadbabaie, and J. M. F. Moura, "On connectivity, observability, and stability in distributed estimation," in Proc. 49th IEEE Conf. Decis. Control, Atlanta, GA, Dec. 2010, pp. 6639-6644.

[22] P. J. Moylan and D. J. Hill, "Stability criteria for large-scale systems," IEEE Trans. Autom. Control, vol. 23, no. 2, pp. 143-149, Apr. 1978.

[23] G. Scorletti and G. Duc, "An LMI approach to decentralized $H_{\infty}$ control," Int. J. Control, vol. 74, no. 3, pp. 211-224, Feb. 2001.

[24] A. Korniienko, G. Scorletti, E. Colinet, and E. Blanco, "Control law design for distributed multi-agent systems," Tech. Rep., Laboratoire Ampère, Ecole Centrale de Lyon, Feb. 2011.

[25] S. Boyd, L. El-Ghaoui, E. Feron, and V. Balakrishnan, Linear Matrix Inequalities in System and Control Theory, SIAM, 1994. 